\title{
Inform-Consult-Empower: A Three-Tiered Approach to eParticipation
}

\author{
Deirdre Lee ${ }^{1}$, Nikolaos Loutas ${ }^{1}$, Elena Sánchez-Nielsen ${ }^{2}$, \\ Esen Mogulkoc ${ }^{3}$, and Oli Lacigova ${ }^{4}$ \\ ${ }^{1}$ DERI, NUI Galway, IDA Business Park, Lower Dangan, Ireland \\ \{Deirdre. Lee, Nikos. Loutas\} @deri.org \\ ${ }^{2}$ Dpto. E.I.O. y Computación, Universidad La Laguna, 38271 Spain \\ enielsen@ull.es \\ ${ }^{3}$ Cyntelix Corporation, Baron van Nagellstr. 136, 3771 LL Barneveld, The Netherlands \\ emogulkoc@cyntelix.com \\ ${ }^{4}$ 21c Consultancy, The Workplace, 105 Ladbroke Grove, Notting Hill, London W111PG, UK \\ olga@21cconsultancy.com
}

\begin{abstract}
While most existing eParticipation projects have embraced the need for citizen engagement to achieve effective democracy, as of yet only limited success has been achieved. This lack of success stems from many challenges and barriers: in some cases, it is a lack of interest in policy issues and low levels of trust in politicians; in others, it is a lack of vision or awareness about the benefits of citizens participation inside the policy-modelling process. This paper describes a three-tiered approach to eParticipation based on a multi-stream policy-making model with three levels of participation: Inform, Consult, Empower. This approach focuses on the level of participation by the user: what are the goals of participation at each of these levels and how do each of these levels of participation relate to current policy-modelling practices. The Puzzled by Policy project will adopt and implement the Inform-Consult-Empower approach, which shows how the social complexity barriers, political culture barriers, technological barriers and organizational structure barriers can be reduced in order to provide effective participation. A use case of how this model will be used to engage Spanish citizens with immigration policy is presented.
\end{abstract}

Keywords: policy-modelling, eParticipation, three-tiered approach, inform, consult, empower.

\section{Introduction}

In current recessionary times the general public's trust in governments is at an alltime low. Attributes such as transparency, openness and accountability are imperative to rebuilding confidence in policy-makers. However for the average citizen, locating, accessing and using up-to-date information, resources and tools can often be very difficult - leaving many citizens unsure about the key regional, national, European and international policymaking issues or even how to begin to understand them. Over the past decade, many governments have attempted to tackle this democratic deficit 
by funding a wide-ranging series of eParticipation projects aiming to broaden and deepen political participation by providing online platforms for citizens to connect with one another and to their elected representatives and governments.

Macintosh [1] characterised three levels of participation that can be used to characterise e-democracy initiates:

- e-enabling (inform) - supporting those who would not typically take advantage of the large amount of public data available,

- e-engaging (consult) - consulting a wider audience to enable deeper contributions and support deliberative debate on policy issues and

- e-empowering (empower) - supporting active participation and facilitating bottom-up ideas to influence the political agenda.

The first wave of eParticipation projects have not been as successful as initially anticipated, with slow uptake from policy actors. This is somewhat surprising, as many studies have investigated and shown that there are many benefits of citizen participation, including tapping into local knowledge and innovation, reducing or avoiding conflict, mobilising new resources including voluntary labour, reducing transaction costs, increasing social inclusion or cohesion and generating trust and social capital [2]. The limitations that have tended to prevent pan-European projects from reaching a mass audience include a lack of interest in policy issues or politics, low levels of trust in politicians, a large and diverse range of policy actors, varying levels of technical skill, a lack of integration of eParticipation strategy into actual government organisation structures, language difficulties and privacy issues [3]. What is evident with many of these barriers is that they are for the most part not technical obstacles, but barriers that apply to participation in the broader sense of the word. The reasons that deter citizens from participating online are common with those that deter citizens from participating offline. This would suggest that there is too much expectation on eParticipation to be the silver bullet of citizen engagement; that there exists a misconception that the use of Information and Communication Technologies (ICT) will alleviate the standard barriers to participation, engage a wide range of citizens and have a direct impact on the policy making-process.

As this has proven not to be the case, the community must reassess eParticipation: what are realistic goals and achievable aims for it? In order to propose how ICT can actually be of benefit to citizens and policy-makers alike, it is important to understand the intricacies of policy modelling itself. In this paper, we revisit the three levels of participation identified by Macintosh and describe how each of them relates to existing policy-modelling processes. In this way, it can be seen that informing, consulting and empowering are each admirable achievements in their own right for an eParticipation platform, in terms of the policy actors involved.

We propose a three-tiered approach, which offers the policy actor all levels of participation in a realistic and achievable setting. The Inform-Consult-Empower approach described in this paper also recognises that citizen engagement is an iterative process; initially policy actors are more likely to want to simply find out information about policies than to discuss them; once informed, policy actors are more likely to want to discuss policy topics than to propose new ideas or drive policy change. At the same time, by providing eParticipation platform designers with a clear outline of the feasible impact each level of participation may potentially have in the policy-making 
process, as well as their limitations, the resultant platforms should not confuse or mislead users in any way. On the contrary, the platforms should be built in such a way to address users' expectant level of participation, while guiding them to a higher level of participation if they so wish. A use-case of the Inform-Consult-Empower approach in action is the 'Puzzled by Policy: Helping you to be part of the EU' project ${ }^{1}$ which aims to provide policy actors with an engaging and easy-to-use platform based on the three-tiered approach presented in this paper, so that they can learn about and engage with policy issues. While the project is not yet mature enough to deliver an overall evaluation of the approach, how the approach is being adopted and implemented in the project will be described.

The remainder of this paper is organised as follows. In section 2, evolutionary and existing policy-modelling paradigms are presented, along with how citizen produced content or knowledge can be utilised. Section 3 provides an overview of barriers and benefits of eParticipation. Section 4 describes the Inform-Consult-Empower approach for eParticipation inline with the multi-stream policy-making paradigm, showing how each of the tiers of this approach relates to a multi-stream paradigm. Section 5 explains how the Inform-Consult-Empower approach will be implemented in the context of Puzzled by Policy. Concluding remarks and future work are provided in Section 6.

\section{Policy Modelling}

Policy formulation is a complex process involving many factors over a potentially long period of time. In the political and social science domain, many models have been proposed to capture the policy inception, implementation and evaluation process. Lasswell's policy stage model [4], also known as the linear model, breaks down the policy process into distinct, sequential stages and is regarded as the 'traditional textbook approach' to policy modelling [5]. The stages that Lasswell defines are: identification of policy problems, agenda setting, formulation of policy proposals, adoption and legitimisation of policies, implementation of policies, and evaluation of a policy's impact.

The policy stage model has been commended, as it emphasises a process of policy making that cuts across and bridges various institutions of governments, it takes policy outcomes into account and, by reducing the intricacies of policy making to manageable analytic units, it has provided an array of useful stage-focused research, particularly regarding agenda setting and policy implementation [6]. However, it has also received criticism from modern-day social scientists, due to the oversimplification of the model into a series of rational steps. Porter and Hicks [5] claim that in the real world, events seldom unfold in this neat, ordered fashion and policy decision making rarely follows this pattern. Another drawback of the stage model is that it assumes that the only actors involved in the policy modelling process are the "policy elites' or those individuals who are considered to be 'government officials' [7]. This is a limiting factor as many instances of policy creation or change actually involve actors external to the official policy or government apparatus.

\footnotetext{
${ }^{1}$ www.puzzledbypolicy.eu
} 
Kingdon [8] proposes an alternative policy model to the stage model based on the garbage-can model: the agenda-setting or multiple-stream model. The multiple-stream model separates the policy-modelling process into the problem stream, policy stream and political stream, as shown in Fig. 1. Kingdon suggest that it is only when these three streams come together at a given point in time, known as the policy window, that policies appear on a government's agenda and have a chance of being implemented.

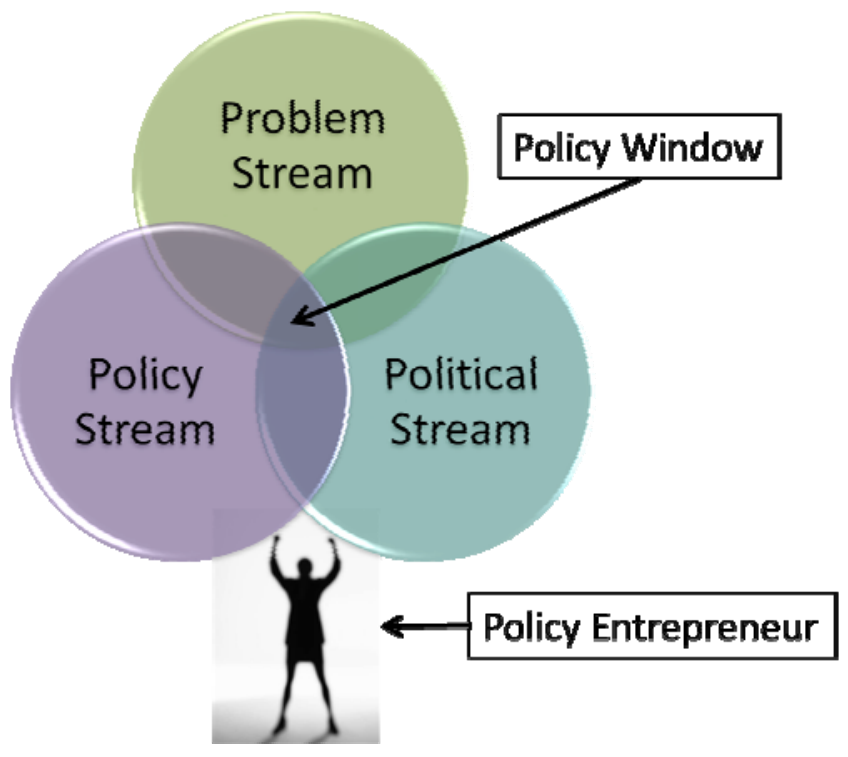

Fig. 1. Multiple Stream Model

The problem stream contains problems, where problems are seen to be social conditions where there is a recognised need for change. Arguments must be made and accepted that a negative social condition is attributable to causes within human control (and not simply a matter of fate or a fact of nature) and amenable to government solution before it can become a problem for public policy [5]. The policy stream (also known as the solution stream) includes potential new ideas that will be tested for feasibility, acceptability and relevance. The multiple-stream model implies the possible existence of policy proposals or solutions, without the existence of a particular problem. In such cases, policy actors may try and identify policy problems to add leverage and support to their proposals, thus pushing these problems onto the agenda. The third component in the multiple stream model is the political stream, which denotes the national mood, organised political interests and the government itself; that is to say the dynamic and often unpredictable elements that will ultimately determine if a problem and a policy alternatives will appear on the government agenda.

According to the multiple stream model, it is only when a problem has been defined, a solution has been identified and the political conditions are right, that the policy window appears, i.e. the time period where the proposed policy is on the 
political agenda, i.e. there is the possibility for an actual policy creation or change. However, should any of these components shift, the window of opportunity may close, for example if key actors change, if the event that caused a change in national mood passes, or if no ready alternative solutions are available.

The final factor in the multiple-stream model is that which actually makes the connections between the three streams: the policy entrepreneur. Most case studies of policy formation pinpoint someone or a small set of people who were central in moving a subject up on the agenda. They are advocates who are willing to invest their resources in return for some anticipated future benefits [5]. Successful entrepreneurs may have expertise or political connections, but it is their persistence and tenacity that more often than not results in their policy of interest getting onto the government agenda. They are ready to strike as soon as a policy window opens and may even encourage the opening of a policy window through strategic promotion and campaigning.

The multiple-stream model is more-widely accepted than the linear approach, as it reflects the loose-coupling of real-world policy making and it accounts for the human element of the process. Running parallel to policy models are many questions. For example who defines the problems, finds the solutions and shapes the political arena? What knowledge are these problems and solutions based on? In the following three sections, we will explore these questions.

\subsection{Policy Actors and Networks}

The human element is at the core of the policy modelling process. While a policy entrepreneur may drive a particular policy change, many individuals, groups and networks contribute to the overall process. Groups that are outspoken and proactive about identifying policy problems, suggesting solutions and attempting to place their views on the agenda table are parties within government, interest groups, researchers, NGOs and the media. These individuals or groups may form networks around particular issues, knowledge-sets or policies.

\subsection{Knowledge Generation}

Knowledge is what shapes the beliefs and drives the decisions of policy actors and networks. Therefore it is important to investigate where and how is this knowledge produced and how is it used in the policy process. Jones [9] highlights the areas presented as key sources of knowledge for policy: research, process, participation and interdisciplinary. Research refers to the creation of high-quality knowledge by experts in the field, for example universities, research institutes, or consultancies. Secondly, Jones presents the process or experience of project and programme implementation as being in itself a source of knowledge for future policy. What is important in this strategy is to monitor and evaluate progress, so that the causal chain of inputs, outputs, outcomes and impacts can be identified and learned from.

The third source of knowledge put forward by Jones, which is related to the central theme of this paper, is citizen participation. He states that there is a great deal of work which advocates for citizens to either be directly involved in generating knowledge for policy or be invited to participate in policy spaces, hence incorporating evidence which reflects their voices on policy issues. While government accountability is 
considered as one of the main grounds for facilitating citizen participation, there are also theories that citizens' experiential knowledge of social issues hold a legitimate and worthwhile perspective and that voicing of that experiential knowledge and personal perspectives bring a new and autonomous discourse to the policy process [10]. On the other hand, the validity of local and indigenous knowledge is often disregarded. Jones also highlights the possibility that engaging citizens may only be tokenistic in nature, or that participatory techniques may serve to reify local culture and reaffirm the agendas of local elites.

Finally Jones presents knowledge generated from multiple and interdisciplinary sources. For complex policy problems, knowledge that is cross-disciplinary and from a variety of sources is required, such as knowledge from those sources mentioned above, combined with values, political judgement, habits and tradition and professional experience and expertise.

\subsection{Knowledge Utilisation}

There has been much research into how and to what extent knowledge, as described in the previous section, is used in policy modelling. In her work on knowledge utilisation and decision-making, Weiss [11] identifies seven models of research utilisation: knowledge-driven; problem-solving; enlightenment; political, tactical; interactive; and intellectual enterprise.

While the knowledge-driven and problem-solving models of research utilisation may seem like logical approaches for knowledge utilisation, they are somewhat inline with the thinking of the policy-stage model described earlier, in that the identification of a social problem or a need for data will precede knowledge generation. However, we have seen that this is often not the case in real-life scenarios, where policymodelling is a complicated and political process. The production of high-quality data does not necessarily induce policy or programme change. Therefore it is the enlightenment model of research that has gained considerable attention and agreement within the knowledge utilisation literature.

Weiss' enlightenment model illustrates the idea that knowledge gained through research can enlighten or broaden the existing knowledge base of policy makers which, over time, can create a gradual shift of conceptual thinking and, therefore, the policies which support that conceptual thinking [7]. We would also argue that, not only policy makers, but the citizens themselves could be more enlightened or 'informed' through existing knowledge. This is a more realistic research utilisation model, as it facilitates a trickle-effect influence of knowledge on the policy process, from many sources, on many actors, over a period of time. We are also of the opinion that the enlightenment model is also inline with Kingdon's multiple-stream model, as the build-up of knowledge through the 'enlightenment' process may at some point induce the opening of a particular policy window.

\section{Citizen Participation and eParticipation}

It is widely accepted that engaging with citizens is not only desired but required for effective democracy and ultimately policy-making. A literature review funded by the Home Office's Civil Renewal Unit [12] found the following sorts of benefits cited for 
participation in policy-making: improved governance, greater social cohesion, improved quality of services, projects and programmes and greater capacity building and learning. Despite these benefits, there remains a slow uptake of citizens to participate in the policy-modelling process. As part of the OECD Focus on Citizens report [3], governments were asked to report a number of reasons for people not wanting to participate in policy making, even when they do not face any particular external barriers. The results are shown in Fig. 3. It was claimed that a staggering $78 \%$ have a low interest in policy and/or politics and $48 \%$ have a low trust in how government uses citizens' input.

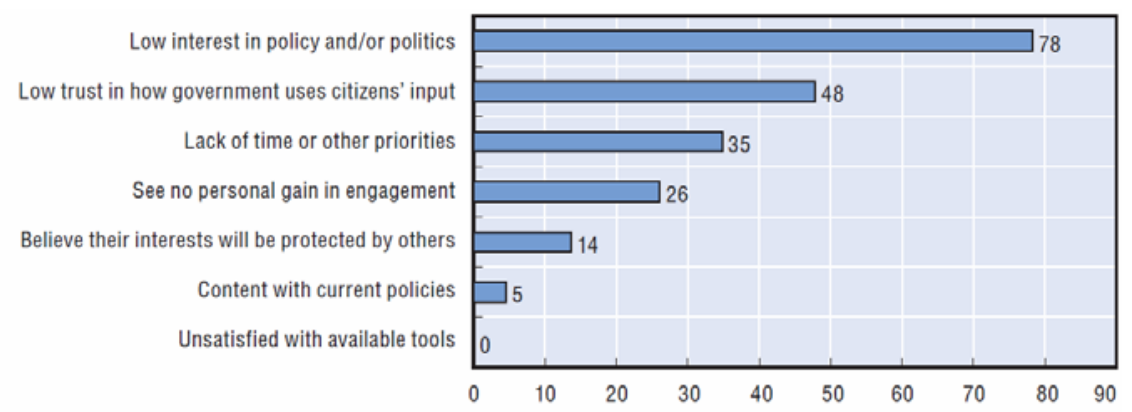

(\% respondents ranking the option as "important" or "very important".)

Fig. 3. Why don't people participate? (\% respondents, $n=25$ countries) [3]

The barriers to citizen participation have also been studied extensively by the eParticipation community. It follows naturally that many of the barriers to offline participation are common with those barriers to online or eParticipation. This is evident from Macintosh's synopsis of the barriers and challenges of eParticipation [13], which are categorised under: social complexity, political culture, organisational structures, technological dependencies, understanding eParticipation.

Social complexity refers to the large and diverse range of policy actors, with varying levels of education, political interest and technical knowledge. There may also exist physical barriers to participation for people, for example physical disabilities, or living in remote, rural areas. Political culture refers to the lack of trust in politicians and governments and a lack of trust that citizens' input will be taken seriously. A lack of commitment from policy-makers in the citizen engagement process helps to fuel this impression of distrust. Organisational structures refers to the lack of integration of eParticipation into routine, government structures and policy processes. Technological dependencies refer to the myth that technologies alone are the silver bullet solution to all citizen participatory problems. In order for eParticipation to be effective, technologies must be integrated into a broader adaptation of government-citizen relationship building. Language difficulties and information management issues are also barriers to adoption. Finally, understanding eParticipation refers to the fact that eParticipation is still a relatively young research area that requires systematic and comprehensive study and evaluation in order to understand its intricacies. 


\section{The Inform-Consult-Empower Approach}

In section 2, an overview of the state-of-the-art of policy modelling and knowledge utilisation in the policy-modelling process was presented. Section 3 provided a brief overview of participation and the main barriers to its adoption. So where does the relatively new discipline of eParticipation fit into the well-established policymodelling field? For many current eParticipation projects, one of the main goals is usually to influence policy-makers and have an impact on policy [14]. However if we look at Weiss' seven meanings of use and Kingdon's multiple-stream model, we see that in real-world policy-modelling processes, it is not as straightforward as generated knowledge immediately forcing a policy change. It is far more likely that knowledge may exist for a period of time, without being linked to a particular policy problem. According to the enlightenment model, knowledge may feed a policy maker's knowledge base, which may ultimately contribute to a shift in conceptual thinking and potentially a change in policy. We build on this theory by proposing that not only does existing knowledge inform policy makers, but it may also inform any actor, group or network with a stake in the policy process.

Based on these findings, in this paper a three-tiered approach is proposed: the Inform-Consult-Empower approach. This approach is inline with the multi-stream policy-making model and, as such, offers policy actors the opportunity to participate in an appropriate and achievable setting. Table 1 describes how each of the tiers in the Inform-Consult-Empower approach relates to the multi-stream model and hence what impact each level of participation can expect to have on the actual policy-making process. As stated previously, the Inform-Consult-Empower approach is structured iteratively: participating at the Inform level is the most straight-forward and requires the least amount of interaction from the user, participating at the Consult level requires more of a time and deliberative commitment from a user, whereas participating at the Empower level requires continuous contribution and in-depth deliberation.

It can be seen that through participation at the Inform tier (e-enabling) policy actors are enlightened by existing knowledge, thus broadening their own understanding of a domain. At this tier, it is also possible to identify what topics are of most interest to users and thus may require policy change, by analysing how a user interacts with the eParticipation platform. These topics may be considered to be in the problem stream. Through participation at the Consult tier (e-engaging), policy actors discuss and deliberate with each other on existing issues (top-down consultation). Frequently visited issues, or those with many contributions, may give an indication of what topics are important to policy actors and are therefore in the problem stream. Through discourse, policy problems may be refined and potential solutions may be discussed and tailored, adding them to the policy stream. Proposed solutions may also be associated with problems that they were not previously associated with. However in order to participate at this tier, the user is expected to have some knowledge, albeit basic, of the knowledge domain. Through participation at the Empower tier (e-empowering), bottom-up, or citizen-initiated, issues and ideas are facilitated. New issues identified by policy actors may be added to the problem stream and new ideas or solutions may be added to the policy stream. Empowered users are very active and may potentially be 
committed to participating in the long-term. This places them in an ideal position to become policy entrepreneurs: those that are central in moving an issue up on the agenda and may even encourage the opening up of a policy window.

Table 1. How the Inform-Consult-Empower approach relates to the multi-stream model

\begin{tabular}{|c|c|c|}
\hline Tier & $\begin{array}{c}\text { Relation to } \\
\text { multi-stream } \\
\text { model }\end{array}$ & Description \\
\hline Inform & 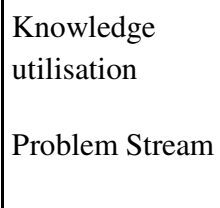 & $\begin{array}{l}\text { Existing knowledge is utilised by stakeholders to broaden their } \\
\text { knowledge base. } \\
\text { Users' behaviour and preferences may be analysed to highlight } \\
\text { popular issues. }\end{array}$ \\
\hline Consult & $\begin{array}{l}\text { Problem Stream } \\
\text { Policy Stream }\end{array}$ & $\begin{array}{l}\text { Popular issues, where there is a potential need for change, are } \\
\text { highlighted through deliberative debate between stakeholders } \\
\text { Potential solutions for these issues are highlighted through } \\
\text { deliberative debate between stakeholders. }\end{array}$ \\
\hline Empower & $\begin{array}{l}\text { Problem Stream } \\
\text { Policy Stream } \\
\text { Policy } \\
\text { Entrepreneur }\end{array}$ & $\begin{array}{l}\text { New discussion topics may be introduced by the user. } \\
\text { New solutions or policy-ideas may be introduced by the user. } \\
\text { Stakeholders may become, or be guided on how to become, } \\
\text { policy entrepreneurs; those who were central in moving a subject } \\
\text { up on the agenda and potentially opening up a policy window. }\end{array}$ \\
\hline
\end{tabular}

The Inform-Consult-Empower approach also serves to address many of the eParticipation barriers categorised by Macintosh [13], (see section 3). Social complexity barriers, such as a large and diverse range of policy actors with varying levels of education, political interest and technical knowledge, are diminished, as there are no requirements on who a user is or what they should know before they participate for the Inform-Consult-Empower approach. Political culture barriers such as a lack of trust in politicians and governments and a lack of trust that citizens' input will be taken seriously may be overridden by the fact that at each of the tiers, involvement of policy-makers themselves is not required. The aim of the approach is to inform policy actors and to add issues to the problem stream and solutions to the policy stream. If there is enough interest and deliberation around a certain topic, the topic may be pushed onto the policy agenda and a policy window may open. Organisational structure barriers refers to the lack of integration of eParticipation into routine, government structures and policy processes. The Inform-Consult-Empower approach directly addresses this barrier, as it is designed inline with current policy-modelling processes, with feasible goals. The approach also alleviates the dependency on technology, by 
focusing the emphasis of eParticipation on the level of participation by the user, what are the goals of participation at each of these levels and how do each of these levels of participation relate to the policy-modelling practices. Finally we believe that structuring eParticipation according to a common approach enables all policy actors to have a better understanding of the goals and limitations of the eParticipation domain.

\section{Implementing the Inform-Consult-Empower Approach: Puzzled by Policy}

The Puzzled by Policy project, which was started in 2010 and will run until 2013, aims to bridge the gap between policy makers and citizens by actively engaging citizens in the policy-making process using the Inform-Consult-Empower approach. The following points outline how the approach described in this paper will be implemented in the Puzzled by Policy project:

- Inform - The 'test where you stand' personal, profiling approach, which became so popular during the Obama campaign and the UK general election in 2010, will be employed so that users will be able to find out where they stand in relation to a specific policy. Hence, an easy-to-use policy profiling application, comprising of a set of questions and answers related to a specific policy will be made available. This will enable users to assess, understand and position their views against the policy in a fun, easy and engaging manner.

- Consult - Puzzled by Policy will facilitate the consultation phase by delivering a visual debating-forum where users can view opinions of other policy actors and, if they wish, contribute to the debate. The online debate will make learning about and debating policy more accessible than ever before.

- Empower - Through collaborative debate, specific topics of interest may emerge, communities may form around issues and policy entrepreneurs may be empowered to petition for that cause. If decision makers are involved in the debate, other stakeholders will be able to give feedback, thus establishing a two way communication channel between citizens and policy makers.

Another important accessibility aspect of the Puzzled by Policy platform is that it utilises widget technology to automatically 'push' the platform to popular social media platforms across Europe. Effectively, this means that we bring the platform to the users rather than trying to attract users to the platform.

\subsection{Case Study: Immigration Policy in Spain}

Designing and preparing a strategic real-life scenario is key to ensuring that the Puzzled by Policy project achieves its goals. In this context, immigration policy in Spain is investigated. The theme of immigration is growing in scope and complexity and is having a serious impact on the European Union and its Member States [15]. Therefore it features prominently on their political agendas. Spain has become a host country ranked tenth in the world in terms of numbers of immigrants and the first source of remittances in Europe, where citizens have already demonstrated their concerns [16]. 
In the years of expansion, immigration policy had a positive impact on Spanish foreign policy: it revitalised bilateral relations and built an image of an open Spain prepared to share its economic growth with citizens of other countries. However, since the burst of the financial crisis, the Spanish government has progressively transformed its immigration policy in order to decrease the numbers of immigrants. This shift has opened a debate [17], which provides an ideal playground for policy actors to express their views and suggest solutions. On this basis, Puzzled by Policy can provide insights and opportunities to actively engage all policy actors. More specifically, the Inform-Consult-Empower approach, upon which Puzzled by Policy capitalises, can be applied in the case of immigration policy debates in Spain as follows:

- Inform: Use Puzzled by Policy's profiling tool to help all policy actors (citizens, policy-makers, researchers, NGOs, immigration associations/observatories and the media) to better understand EU immigration policies and find out what particular policies mean to them on the national level.

- Consult: Use Puzzled by Policy's visual debate forum to debate and share opinions on hot immigration policies, such as 'the voluntary return plan', thus giving voice to minority views and drawing out unspoken opinions.

- Empower: Use Puzzled by Policy's visual debate forum to connect all policy actors, thus helping decision makers at both the national and EU level to understand the impact of their decisions on society.

\section{Conclusions and Future Work}

This paper described a three-tiered approach to eParticipation based on a multi-stream policy-making model with three levels of participation: Inform, Consult, Empower. The Inform-Consult-Empower approach focuses on the level of participation by the policy actors. Different types of policy actors have different goals and benefit differently from their engagement in any of the three tiers. The Puzzled by Policy project is implementing the Inform-Consult-Empower approach in order to promote social inclusion and mass civic engagement in policy making through the delivery of the Puzzled by Policy platform. A policy profiling application and visually-enabled debate forum will allow connect policy actors and will enable the average user to be informed (Inform), share knowledge and opinions (Consult) and pursue a particular issue (Empower). The use of widgets will allow users to participate through their favourite social media platforms, thus removing technological barriers and steep learning curves. Concluding, the experience gained during the design and implementation of the Inform-Consult-Empower approach reveals that the main challenges to be faced are not technical, but rather socio-political. In this context, Puzzled by Policy engages the policy actors from a very early stage in the design and the development of the Puzzled by Policy platform to maximise the platform's uptake and ensure its sustainability beyond the scope of the project.

Acknowledgments. The work presented in this paper has been funded in part by the European Union under Grant No. 256261 (Puzzled by Policy - CIP-ICT-PSP-20093bis) and by Science Foundation Ireland under Grant No. SFI/08/CE/I1380 (Lion-2). 


\section{References}

1. Macintosh, A.: Characterizing E-Participation in Policy-Making. In: 37th Annual Hawaii International Conference on System Sciences (HICSS), Hawaii, US (2004)

2. Smith, S.: D1.3c: Main benefits of eParticipation developments in the EU - a contextualisation with reference to the EU governance regime and the European public sphere. Study and supply of services on the development of eParticipation in the EU, ed. European eParticipation (2009)

3. OECD: Focus on Citizens: Public Engagement for Better Policy and Services: OECD Studies on Public Engagement (2009)

4. Lasswell, H.D.: The Policy Orientation. In: Lasswelland, H.D., Lemer, D. (eds.) The Policy Sciences. Stanford University Press, Stanford (1951)

5. Porter, R.W., Hicks, I.: Knowledge Utilization and the Process of Policy Formation: Toward a Framework for Africa. SARA Project: USAID (1995)

6. Sabatier, P.A., Jenkins-Smith, H.C.: Policy Change and Learning: An Advocacy Coalition Approach. Westview Press, Boulder (1993)

7. Nielson, S.: IDRC-Supported Research and its Influence on Public Policy Knowledge Utilization and Public Policy Processes: A Literature Review. Evaluation Unit, IDRC (2001)

8. Kingdon, J.W.: Agendas, Alternatives and Public Policies. Addison-Wesley Educational Publishers, Reading (1984)

9. Jones, H.: State-of-the-art Literature Review on the Link between Knowledge and Policy in Development, Working Paper No. 5: Overseas Development Institute (ODI) IKM Emergent (2009)

10. Morgan, M.S.: Voice and the facts and observations of experience. Working papers on the nature of evidence: how well do 'facts' travel?: Department of Economic History, London School of Economics and Political Science, London, UK (2008)

11. Weiss, C.H.: Policy research as advocacy: Pro and con. Knowledge, Technology \& Policy 4(1), 37-55 (1991)

12. Involve: The True Costs of Public Participation: Involve (2005)

13. Macintosh, A.: Challenges and barriers of eParticipation in Europe? In: Forum for the Future of Democracy, Riksdag, Stockholm (2007)

14. OECD: Engaging Citizens in Policy-making: Information, Consultation and Public participation. OECD Public Management Policy Brief (2001)

15. Sides, J., Citrin, J.: European Opinion about Immigration: the Role of Identities, Interests and Information. British Journal of Political Science 37, 477-504 (2007)

16. Balch, A.: Economic Migration and the Politics of Hospitality in Spain: Ideas and Policy Change. Journal Politics and Policy 38(5), 1037-1065 (2010)

17. Tedesco, L.: Immigration and Foreign Policy: The Economic Crisis and its Challenges. FRIDE (2010) 